\title{
A NEW MULTI-BODY DYNAMIC MODEL FOR SEAFLOOR MINER AND ITS TRAFFICABILITY EVALUATION
}

\author{
Dai, Y. ${ }^{* * * * \# ; ~ Z h u, ~ X . * ; ~ C h e n, ~ L . ~ S . ~ ; ~ L i u, ~ H . * ; ~ Z h a n g, ~ T . ~}{ }^{*} \&$ Liu, S. J. .** \\ * College of Mechanical and Electrical Engineering, Central South University, Changsha 410083, China \\ ${ }^{* * *}$ Shenzhen Research Institute, Central South University, Shenzhen 518000, China \\ E-Mail: daiyu_6@aliyun.com (" Corresponding author)
}

\begin{abstract}
In this paper, the particular mechanical interaction relationships between a seafloor tracked miner and the sediment are obtained through laboratory-simulated experiments. Underwater sediments exhibit entirely different shear properties compared to land surface soils. An innovative user-written subroutine for characterizing the mechanical models of various soil types is compiled and linked to the RecurDyn simulation environment and then validated against an available commercial program and with laboratory tests. A new three-dimensional multi-body dynamic simulation model of the miner is developed in RecurDyn by integrating the developed mechanical model of the seafloor sediment. Simulations are conducted to measure the miner's geometric trafficability and locomotion performance. Additionally, a mathematical model to evaluate the miner's tractive trafficability in relation to its slip ratio and its primary design parameters is established, and the results demonstrate that there is an optimum slip ratio that produces a maximum traction force for the miner. This research provides a valuable and effective simulation modelling method for trafficability, locomotion predictions, design optimization and motion control of tracked-operated vehicles under various terrain conditions.

(Received, processed and accepted by the Chinese Representative Office.)
\end{abstract}

Key Words: Seafloor Tracked Miner, Track-Sediment Interaction Mechanics, 3D Multi-Body Dynamic Model, Simulation Analysis, Trafficability Evaluation

\section{INTRODUCTION}

Mining of deep seafloor mineral resources has become an international focal point and is likely to be commercially implemented within the next five years. The seafloor miner is a cutting edge technology and a crucial subsystem of the integrated ocean mining system. Its trafficability and locomotion performance directly affects the continuous operation performance of the entire system.

To evaluate the trafficability of tracked vehicles on the seafloor, Schulte et al. established a lab device to conduct bearing and shear tests in a bentonic-water mixture and to calculate the static and dynamic sinkages [1-2]. Yamazaki et al. presented the geotechnical properties of the deep seafloor sediments, including the friction angle, cohesion, elasticity and viscosity modules, as well as the examined mechanical interactions with a seafloor miner [3]. Choi et al. studied the probable distributions of the shear strengths of seafloor sediments based on samples obtained by multiple corers and applied one of these distributions to design a seafloor miner [4]. Choi et al. also performed traction performance experiments of a small-scale tracked vehicle in a lab soil bin and investigated the influences of its design parameters on the trafficability [5].

To study dynamics and analyse locomotion, Kim et al. developed a novel method using the Euler parameters to dynamically analyse a tracked vehicle on the seafloor [6] and further proposed a subsystem synthesis method to analyse a multi-body miner [7]. Dai and Liu proposed and built a seafloor miner as a single-body vehicle model with mesh elements to track sediment interactions and carried out fast simulations of the miner and the complete ocean mining system [8]. In addition, research has been performed modelling tracked vehicles on land surface soils. Rubinstein et al. used the LMS-DADS simulation code to develop a 3D 
multi-body model of tracked off-road vehicles and applied user-written forces to represent the track-terrain interactions [9, 10]. Gao and Wong (and others) developed two different dynamic simulation models, known as NTVPM and RTVPM, to parametrically model, analyse and evaluate various tracked vehicles with flexible tracks and long-pitch tracks [11-13]. Janarthanan et al. developed a simulation model for analysing the longitudinal dynamics of a heavy-type tracked vehicle and verified this model by comparing the predicted values with test data [14].

So far, however, the unique mechanical interactions between a seafloor miner and the sediment have not yet been ascertained quantitatively; therefore, a mathematical model for evaluating a miner's trafficability has yet to be established. In addition, neither the development of a complex 3D multi-body dynamic simulation model for a tracked miner nor a mechanical model of the seafloor sediment integrated into a simulation model has been realized. Given these deficiencies, the corresponding research was conducted.

\section{MECHANICAL INTERACTION EXPERIMENTS}

As ocean in-situ mechanical tests between the seafloor miner and the sediment are extremely difficult and costly to perform, laboratory-simulated experiments were conducted. The sediment layer at 15-20 cm below the seafloor surface is regarded as the optimal bearing and traction layer for a miner; this layer's in-situ physical and geotechnical characteristics were referred to when preparing the simulated sediment. A bentonite-water mixture was considered to be the most suitable substitute [1-4]. When the experimental data approached or matched the in-situ data well, the simulated sediment was considered to be roughly prepared.

Fig. 1 illustrates the experimental apparatus, including the soil bin, the simulated shear plates, the penetration plates, the hydraulic driving system, the pull-pressure sensor and the data acquisition system. The shear plates and the penetration plates were driven by the hydraulic system, while, simultaneously, the pressure and shear force were measured by the high-precision sensors.

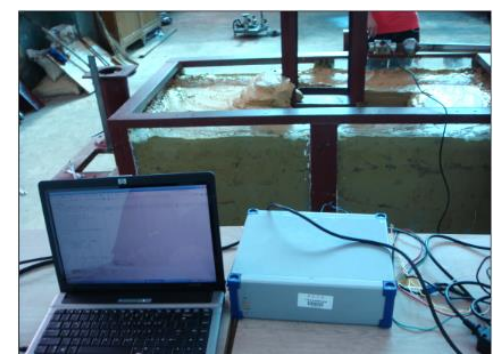

a)

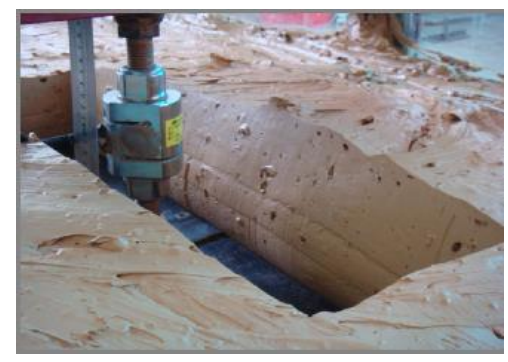

b)

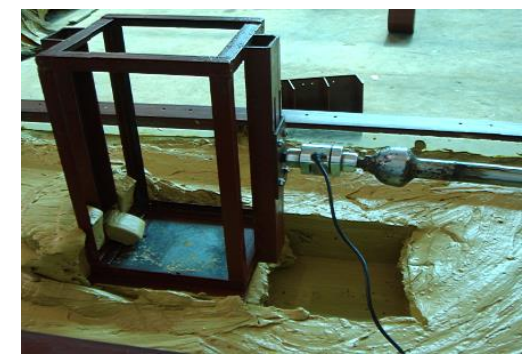

c)

Figure 1: Photographs of the mechanical experiments: a) total experiment apparatus; b) penetration experiment; and c) shear experiment.

A series of experiments was conducted with different sized penetration plates and shear plates. Correspondingly, pressure-sinkage and shear stress-displacement experimental curves were obtained, as shown in Fig. 2, and were approximately fit with polynomial curves.

The pressure-sinkage experimental curves can be characterized by Bekker's exponential equation as follows [15]:

$$
p=\left(\frac{k_{c}}{b}+k_{\phi}\right) z^{n}
$$

where $p$ is the normal pressure; $b$ is the track's width; $k_{c}$ and $k_{\phi}$ are the soil cohesive and friction moduli, respectively; $z$ is the sinkage; and $n$ is the soil deformation exponent. 

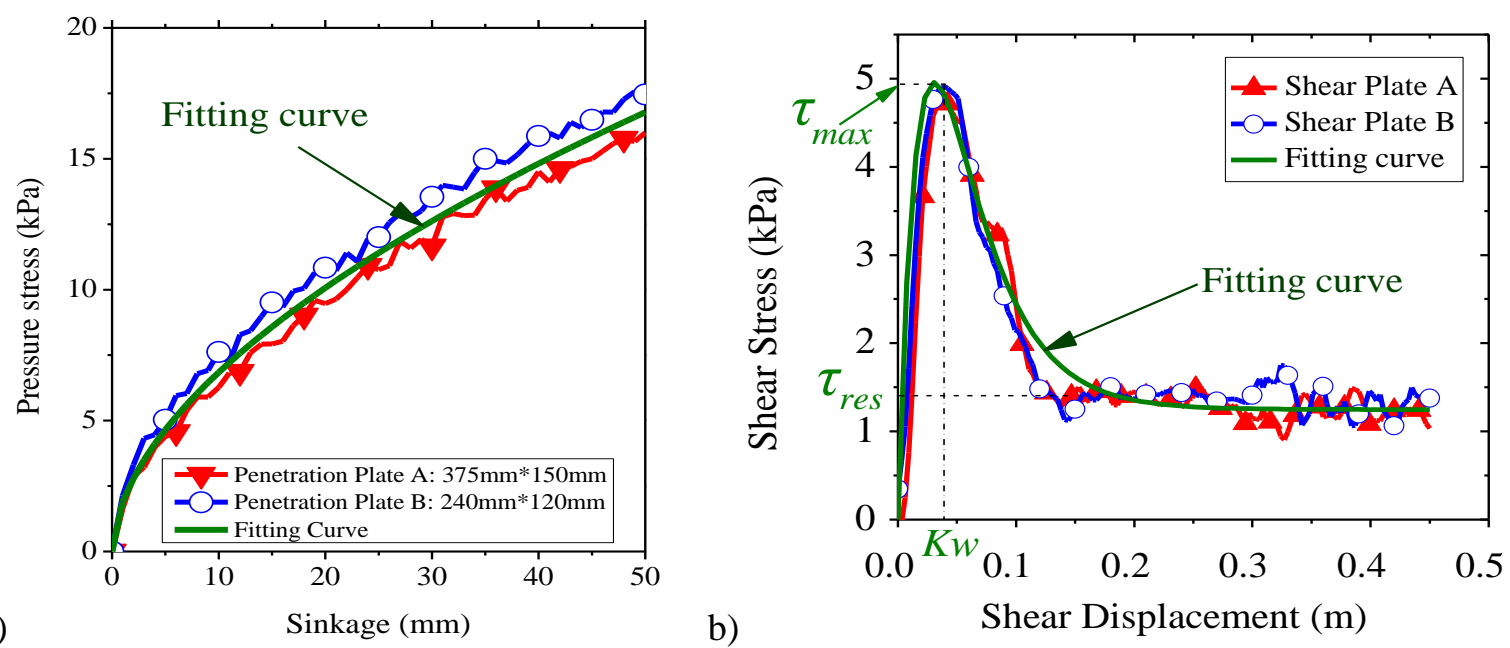

Figure 2: Laboratory experimental curves and fitting curves: a) pressure-sinkage; b) shear stressdisplacement.

The shear property demonstrates that the shear stress increases sharply initially, reaches a peak value at a specific shear displacement, and then decreases and approximates a constant residual value with continuously increasing shear displacement. This is entirely different for land surface soils, as described by Janosi and Hanamto's equation [16]. However, Wong's equation appears to be a good fit [17]:

$$
\tau=\tau_{\max } \cdot K_{r} \cdot\left\{1+\left[\frac{1}{K_{r}\left(1-e^{-1}\right)}-1\right] e^{1-j / K_{\omega}}\right\} \cdot\left(1-e^{-j / K_{\omega}}\right)
$$

where $\tau_{\max }$ is the maximum shear stress, $K_{r}$ represents the ratio of the residual shear stress to $\tau_{\max }, j$ is the shear displacement, and $K_{\omega}$ is the shear displacement at $\tau_{\max }$.

Processing the experimental data reveals the following key parameters to describe the pressure-sinkage and shear stress-displacement relationships for a given sediment layer (Table I). The corresponding fitting curves are plotted in Fig. 2.

Table I: Key mechanical parameters of the seafloor sediment.

\begin{tabular}{|l|c|c|}
\hline \multicolumn{1}{|c|}{ Parameters } & Symbols & Values \\
\hline Cohesive modulus $\left(\mathrm{N} / \mathrm{m}^{\mathrm{n}+1}\right)$ & $k_{c}$ & $1.62 \times 10^{3}$ \\
\hline Friction modulus $\left(\mathrm{N} / \mathrm{m}^{\mathrm{n}+2}\right)$ & $k_{\phi}$ & $7.71 \times 10^{4}$ \\
\hline Deformation exponent & $n$ & 0.56 \\
\hline Maximum shear stress $(\mathrm{MPa})$ & $\tau_{\max }$ & 4.9 \\
\hline Residual shear stress $(\mathrm{MPa})$ & $\tau_{r e s}$ & 1.4 \\
\hline Ratio of residual shear stress to maximum shear stress & $K_{r}$ & 0.28 \\
\hline Shear displacement corresponding to the maximum shear stress $(\mathrm{m})$ & $K_{\omega}$ & 0.035 \\
\hline
\end{tabular}

\section{MULTI-BODY DYNAMIC SIMULATIONS OF A SEAFLOOR MINER}

\subsection{Multi-body model of a miner with a user-written sediment mechanical subroutine}

The RecurDyn/Track program, based on a relative coordinate system and a recursive algorithm, is used to establish a new 3D multi-body dynamic simulation model of a seafloor tracked miner, as shown in Fig. 3. Table II lists its structural parameters. 


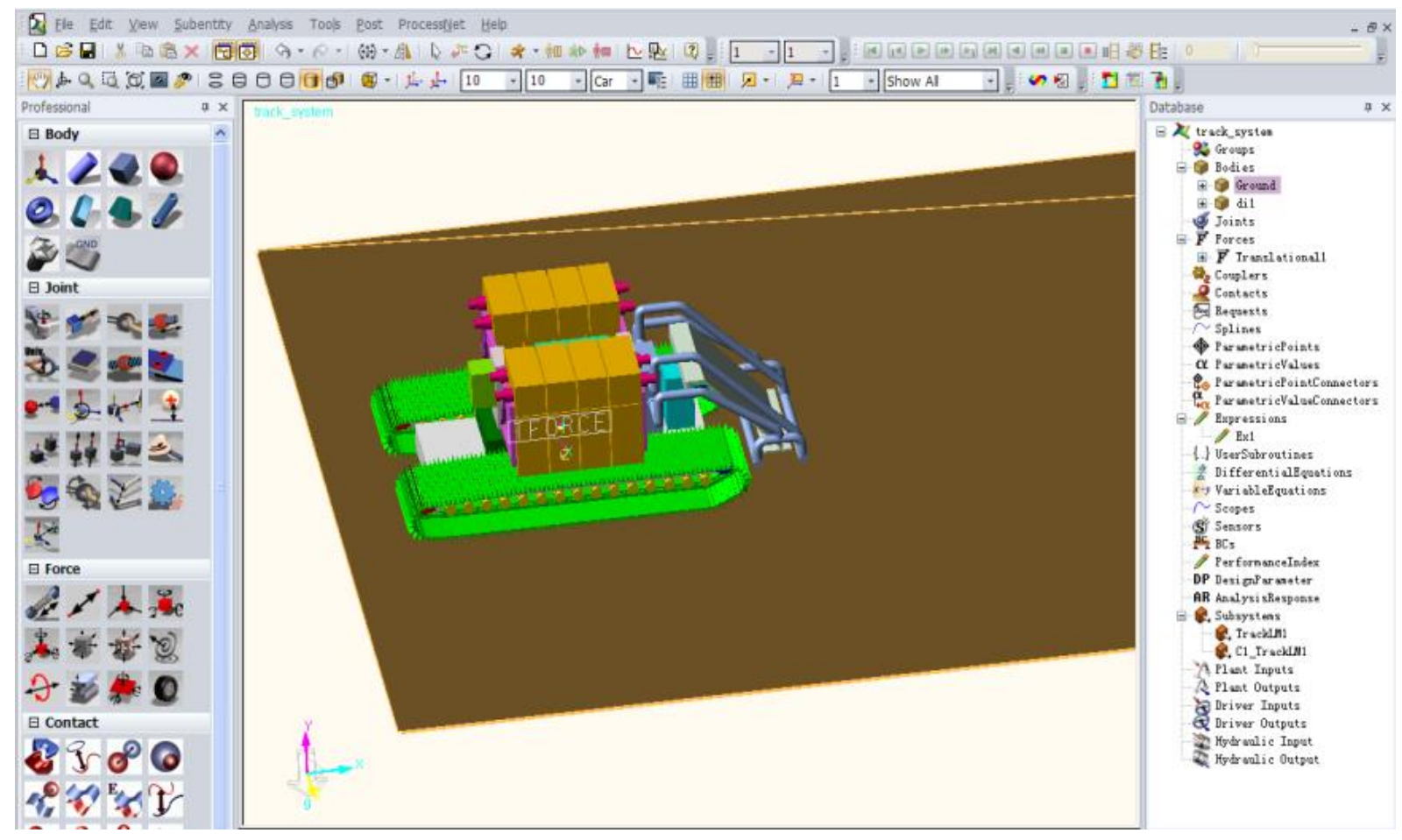

Figure 3: A 3D multi-body dynamic simulation model of a seafloor tracked miner.

Table II: Structural parameters of the miner.

\begin{tabular}{|l|c|}
\hline \multicolumn{1}{|c|}{ Parameters } & Values \\
\hline Total weight in air (tons) & 32 \\
\hline Total weight underwater (tons) & 1.17 \\
\hline Overall dimension $(\mathrm{m})$ : Length $\times$ width $\times$ height & $9.2 \times 5.2 \times 3$ \\
\hline Track contact length $(\mathrm{m})$ & 6.2 \\
\hline Track width $(\mathrm{m})$ & 1.7 \\
\hline Distance between centre lines of tracks $(\mathrm{m})$ & 3.5 \\
\hline Track pitch $(\mathrm{m})$ & 0.15 \\
\hline Grouser height $(\mathrm{m})$ & 0.13 \\
\hline Diameter of road wheel $(\mathrm{m})$ & 0.2 \\
\hline Number of road wheels per track & 15 \\
\hline
\end{tabular}

It is important to note that current commercial simulation programs for tracked vehicles provide only one type of soft soil mechanical model, known as Bekker's pressure-sinkage relationship, and Janosi and Hanamto's shear stress-displacement relationship. However, this type of soft soil model is not appropriate for expressing the special characteristics of the seafloor sediment.

An innovative user-written subroutine for characterizing the mechanics of various soils was developed using the $\mathrm{C}$ language in the Visual Studio.Net environment, as shown in Fig. 4. The dynamic link library (DLL) created in Visual Studio.Net was then called by RecurDyn/Track to allow this particular mechanical model of the seafloor sediment to be integrated into the RecurDyn/Track system. 


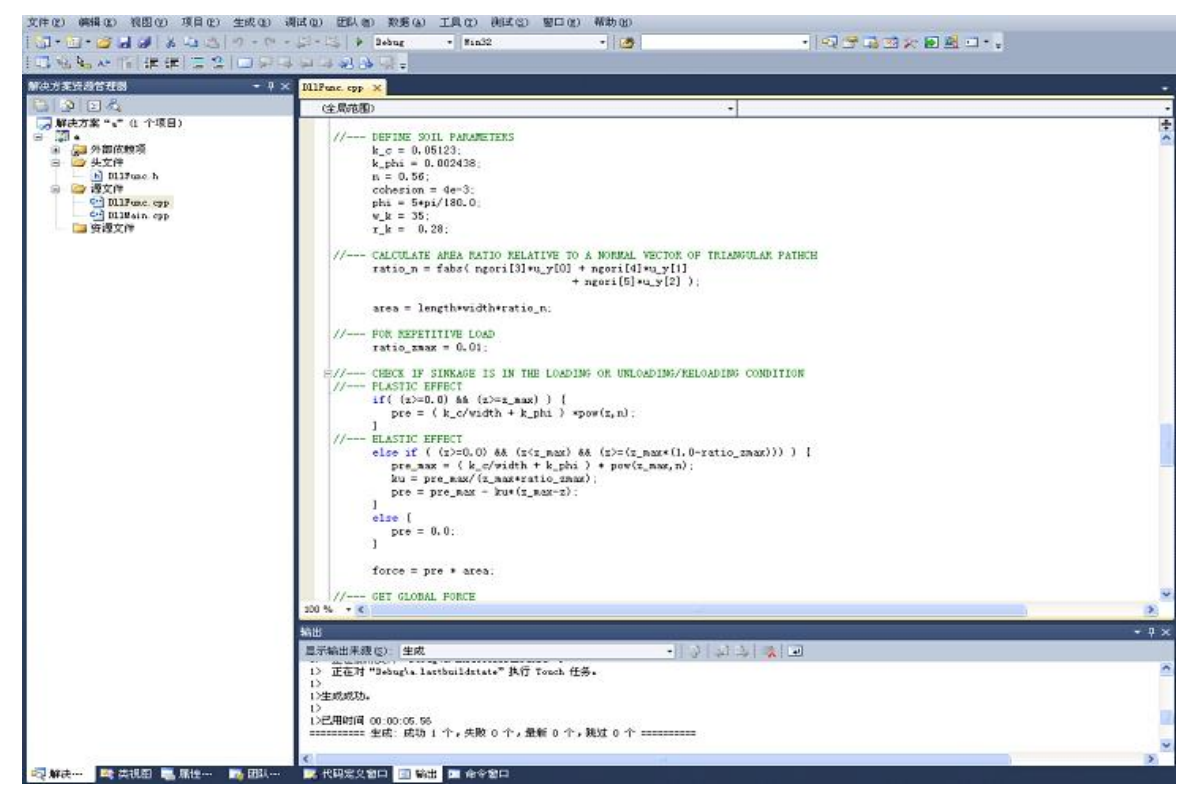

Figure 4: User-written subroutine development interface in Visual Studio.Net for the mechanical model of the seafloor sediment.

\subsection{Simulation validation}

Fig. 5 compares simulations of the user-written subroutine model and the provided commercial program model for the same tracked vehicle and the same typical land soil under various motion conditions. The simulation results of the user-written subroutine model are consistent with those provided by the commercial program model.

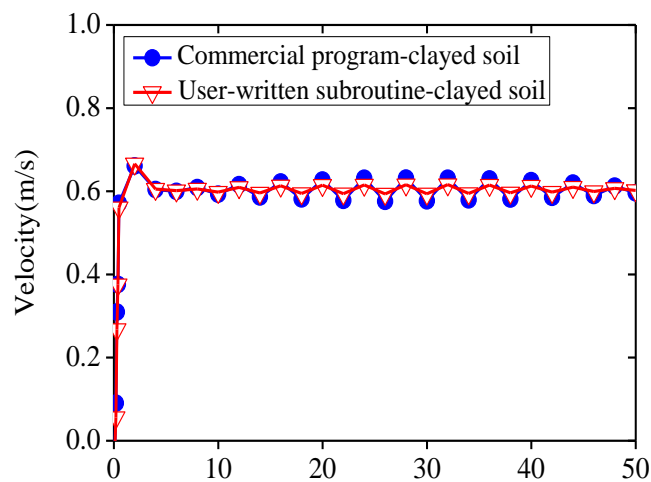

a)

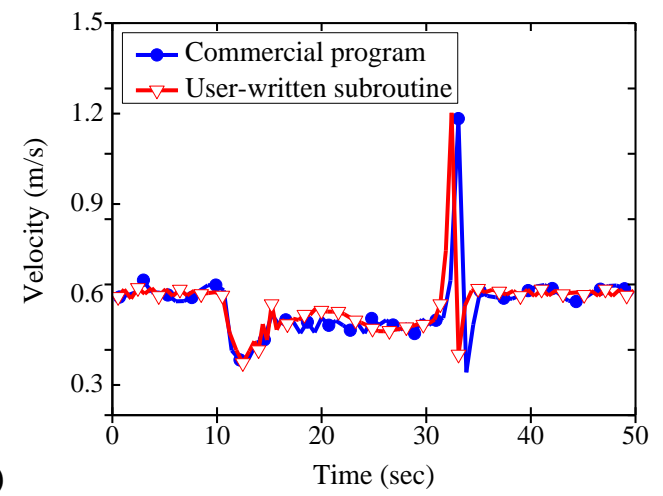

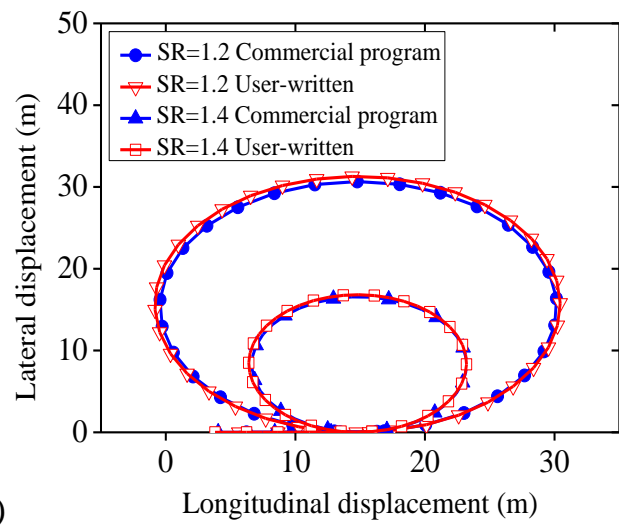

b)

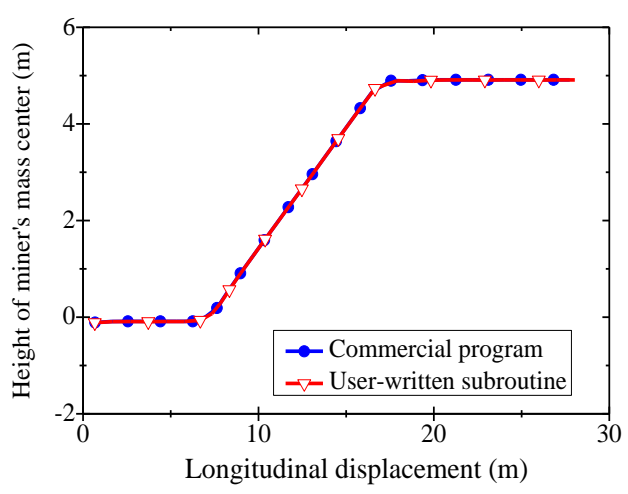

Figure 5: Simulation comparisons of the user-written subroutine model and the provided commercial program model: a) velocities on flat ground; b) turning trajectories; c) climbing velocities; and d) vertical positions for climbing. 


\subsection{Lab experiment validation}

A small tracked vehicle was manufactured for trafficability and locomotion research on soft terrain, as shown in Fig. 6 a. Correspondingly, a 3D multi-body dynamic simulation model was built using the above-proposed method with the developed user-written soil mechanical subroutine. The lab field terrain was dry sand.

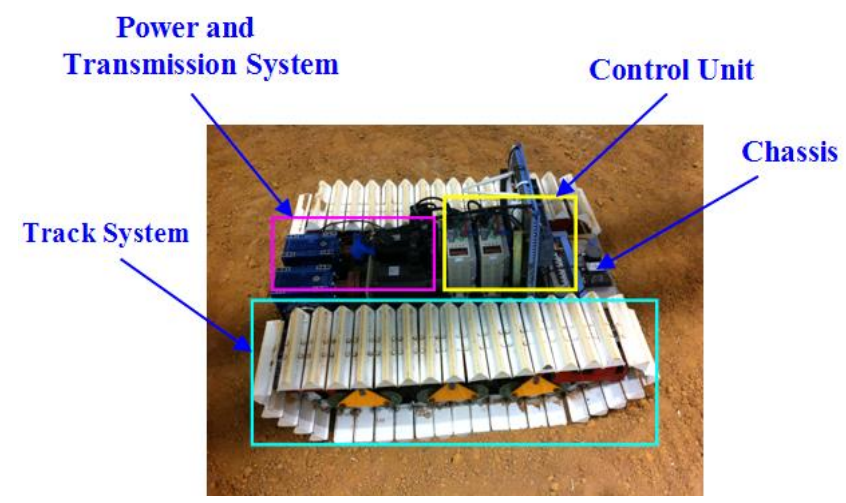

a)

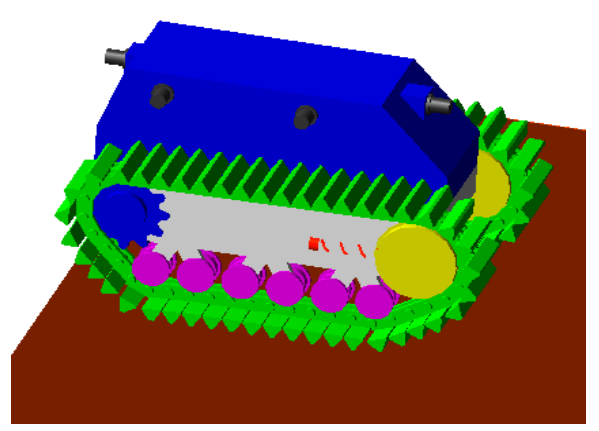

b)

Figure 6: Laboratory tracked test vehicle: a) photograph; b) 3D multi-body simulation model.

The vehicle utilized two $1.2 \mathrm{~kW}$ permanent magnet synchronous servo motors to drive the left and right tracks independently. The servo motors were equipped with absolute optical encoders. Each motor's speed was controlled by a servo driver, which transmitted velocity and torque information to the computer, which was a Siemens S7-1200 PLC module. A Honeywell 3-axis digital compass HMR3000 was installed on the chassis to measure the heading, pitch and roll angles. In addition, a single axis gyroscope was installed to measure the turning angular velocity, and a single axis acceleration sensor was used to measure the acceleration.

For the turning experiment, the input velocities for the inner and outer tracks were, respectively, set to $0.05 \mathrm{~m} / \mathrm{s}$ and $0.16 \mathrm{~m} / \mathrm{s}$. Fig. 7 presents the vehicle's trajectory and turning angular velocities. The field test result showed good agreement with the simulation result.
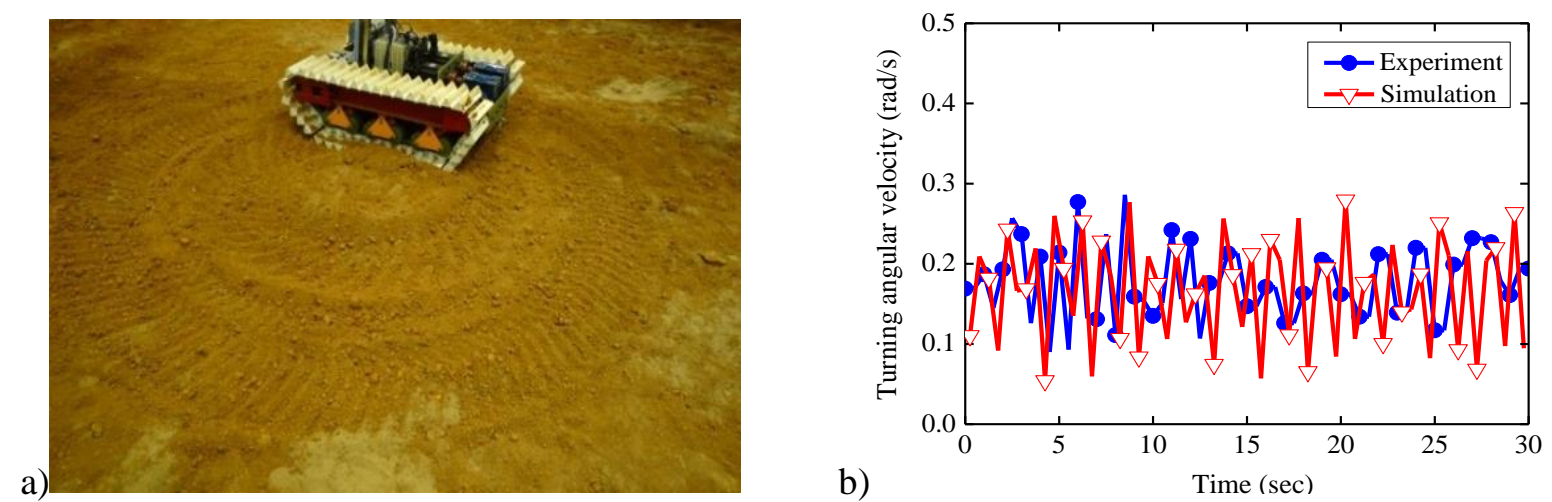

Figure 7: Turning experiment and simulation of a tracked vehicle: a) trajectory photograph; b) turning angular velocities.

For the climbing experiment, the lab field slope was approximately 26.5 degrees and the input velocity for the vehicle was set to $0.1 \mathrm{~m} / \mathrm{s}$. Fig. 8 compares the pitch angles between the experiment and the simulation. The changes in the observed trends matched each other well, in spite of differences in the position of the centre of mass between the simulation model and the real vehicle. 


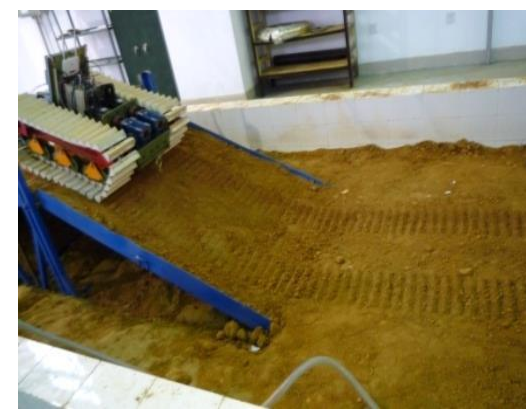

a)

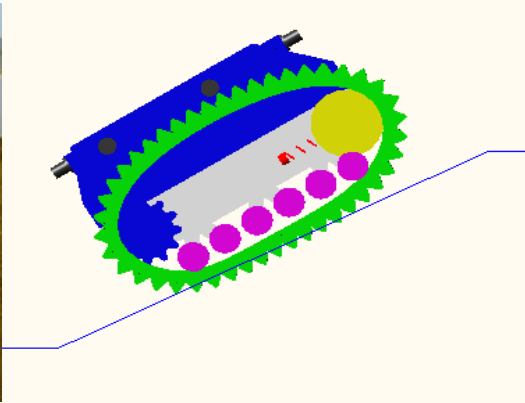

b)

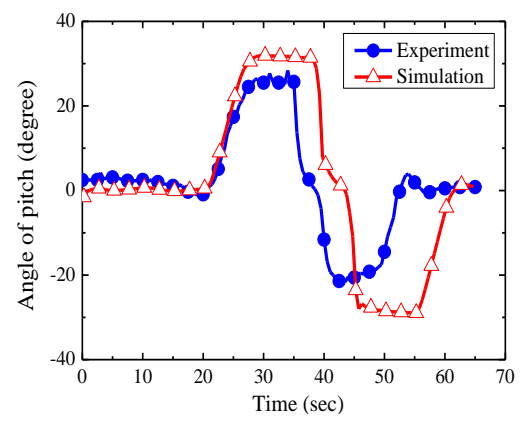

c)

Figure 8: Climbing experiment and simulation of a tracked vehicle: a) photograph of the experiment; b) simulation model; and c) pitch angle.

These results show that the accuracy of the simulations are adequate and that this method can be used effectively to develop a multi-body simulation model of a seafloor tracked miner given the special characteristics of the seafloor sediment.

\subsection{Dynamic simulations of the miner under various working conditions}

Fig. 9 a illustrates the straight-line motion simulation of the miner with an input velocity of $0.6 \mathrm{~m} / \mathrm{s}$ on a homogeneous sediment. Its average sinkage was approximately $55 \mathrm{~mm}$. As the grouser height of the miner was $130 \mathrm{~mm}$, the grouser top could reach a sediment layer depth of $185 \mathrm{~mm}$, which coincides with the optimum traction and bearing sediment layer depth of 150 to $200 \mathrm{~mm}$ for the miner, providing it with good bearing trafficability. Fig. $9 \mathrm{~b}$ presents the motion trajectory in the case where the sediment's shear strength under both of the miner's tracks was different, while the input velocities for the tracks were the same. An apparent trajectory deviation occurred because of the different mechanical properties that led to the different traction forces under the tracks; this produced a turning moment, causing the miner to deviate from its desired path. Trajectory deviations are undesirable and will affect the mining efficiency and safety. According to regulations, the permissible deviation for a miner should not exceed $\pm 1 \mathrm{~m}$. Therefore, the actual motion states of the miner need to be well controlled to follow its predetermined path in the face of uncertain or adverse seafloor environments.
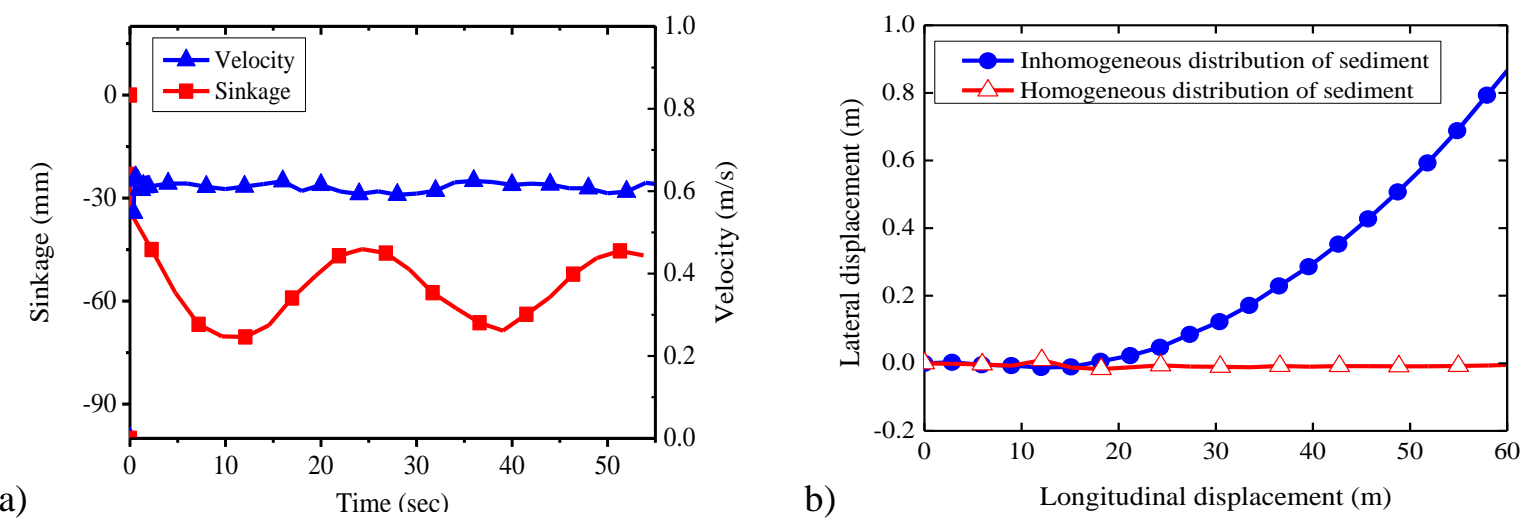

Figure 9: Longitudinal straight-line motion simulations: a) velocity and sinkage simulation curves on the homogeneous sediment; b) compared motion trajectories on the homogeneous and inhomogeneous sediments.

The ideal turning velocity ratio for the outer and inner tracks of a miner on the seafloor should not exceed 1.4 to ensure safety. A ratio of 1.2 requires a velocity of $0.5 \mathrm{~m} / \mathrm{s}$ for the inner track. Fig. 10 compares turning trajectory simulations on the seafloor sediment and on land in a typical clayed soil. The turning radius on the seafloor is approximately $75 \mathrm{~m}$, while 
on the terrestrial clayed soil, it is approximately $40 \mathrm{~m}$. A slip phenomenon obviously occurs during locomotion on the seafloor. Therefore, stricter requirements are necessary for trajectory planning and control for a miner on the seafloor.
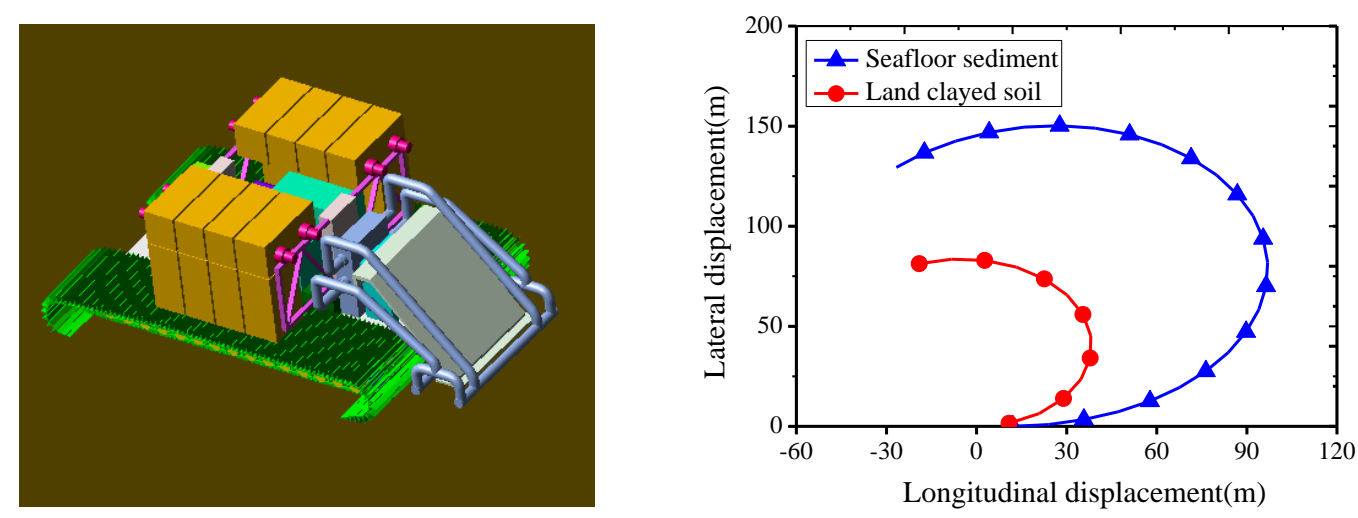

Figure 10: Turning trajectory simulations for the miner on the seafloor sediment and on typical land soil.

Following the topography and geomorphology characteristics of the seafloor, as associated with the obstacle-crossing requirements for a miner, an obstacle-crossing simulation was developed, including a slope of less than 15 degrees, a slope height of less than $0.5 \mathrm{~m}$ and a ditch width of less than $1 \mathrm{~m}$. The virtual topography model of the seafloor was secondarily developed in RecurDyn/Track.

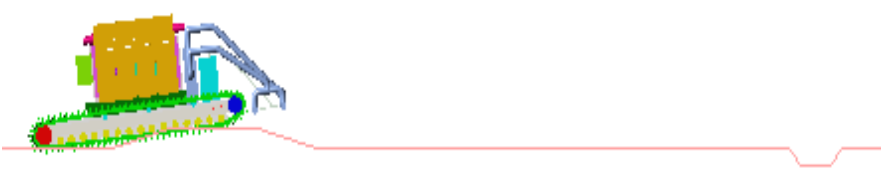

a)

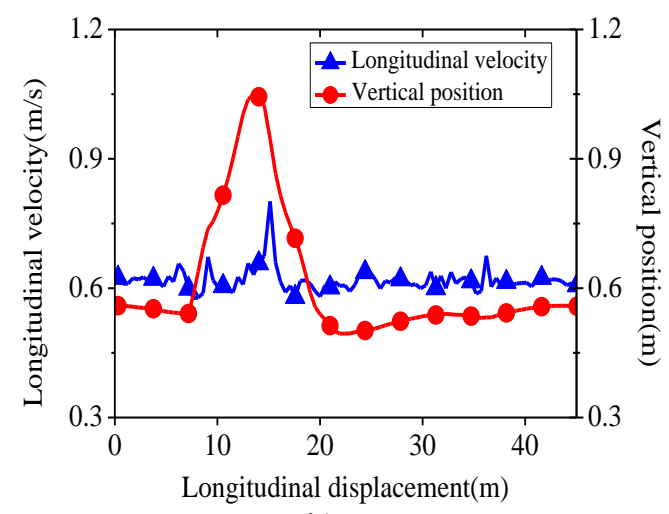

b)

Figure 11: Obstacle-crossing simulations of the miner: a) simulation interface; b) simulation curves.

The simulation reflects the actual motion states of the miner. We conclude that the climbing and striding abilities of the miner on the seafloor can satisfy the safety requirements very well and that the miner has good geometric trafficability and locomotion.

\section{MATHEMATICAL MODEL FOR THE TRACTIVE}

\section{TRAFFICABILITY EVALUATION}

\subsection{Total shear force}

Applying the experimental shear stress-displacement relationship and integrating the shear stress over the whole track-sediment contact area, the total shear force developed beneath the tracks of the miner can be written as:

$$
F_{t}(i)=b \int_{0}^{l}(c+p(x) \tan \phi) K_{r}\left\{1+\left[\frac{1}{K_{r}\left(1-e^{-1}\right)}-1\right] e^{1-i x / K_{\omega}}\right\}\left(1-e^{-i x / K_{\omega}}\right) d x
$$


where $c$ is the sediment cohesion; $\phi$ is the sediment internal friction angle; $b$ and $l$ are the width and length of the track, respectively; $i$ is the slip ratio; and $x$ is the distance relative to the origin of the track-sediment interface.

In practice, it is generally regarded that the normal pressure distribution is seldom uniform; the multi-peak pressure distribution characteristic, as follows, is the most proximate case:

$$
p=\frac{W}{2 b l}\left(1+\cos \frac{2 n_{p} \pi x}{l}\right)
$$

where $n_{p}$ is the number of periods.

Substituting Eq. (4) into Eq. (3), the following shear force, as developed beneath the tracks, can be obtained:

$$
\begin{aligned}
& F_{t}(i)=2 b \int_{0}^{l}\left(c+\frac{W}{2 b l}\left(1+\cos \frac{2 n_{p} \pi x}{l}\right) \tan \phi\right) K_{r}\left\{1+\left[\frac{1}{K_{r}\left(1-e^{-1}\right)}-1\right] e^{1-i x / K_{\omega}}\right\}\left(1-e^{-i x / K_{\omega}}\right) d x \\
& =(A c+W \tan \phi) K_{r}\left\{1+\frac{K_{\omega}}{i l}\left(e^{-\frac{i l}{K_{\omega}}}-1\right)-\frac{K_{\omega}}{2 i l}\left[\frac{1}{K_{r}\left(1-e^{-1}\right)}-1\right]\left(2 e^{-\frac{i l}{K_{\omega}}}-e^{1-\frac{2 i l}{K_{\omega}}}-e\right)\right\}-(W \tan \phi) K_{r} \frac{K_{\omega}}{2 i l}\left[\frac{2\left(e^{1-\frac{i l}{K_{\omega}}}-e+e^{-\frac{i l}{K_{\omega}}}-1\right)}{1+\frac{4 n_{p}{ }^{2} \pi^{2} K_{\omega}{ }^{2}}{i^{2} l^{2}}}+\frac{e^{1-\frac{i l}{K_{\omega}}}-e}{1+\frac{2 n_{p}{ }^{2} \pi^{2} K_{\omega}{ }^{2}}{i^{2} l^{2}}}\right]
\end{aligned}
$$

The shear force developed along the lateral surfaces of the grousers should be taken into account and can be computed by referring to the above equation.

Finally, the total shear force obtained is:

$$
\begin{aligned}
F_{S}(i)= & F_{t}(i)+F_{g}(i) \\
= & \left\{(A+4 h l) c+W \tan \varphi\left[1+0.64 \frac{h}{b} \arctan \left(\frac{h}{b}\right)\right]\right\} K_{r}\left\{1+\frac{K_{\omega}}{i l}\left[e^{-\left(i l l / K_{\omega}\right)}-1\right]-\left[\frac{e}{K_{r}(e-1)}-1\right] \frac{K_{\omega}}{2 i l}\left[2 e^{1-\left(i l / K_{\omega}\right)}-e^{1-\left(2 i l / K_{\omega}\right)}-e\right]\right\} \\
& -W\left\{1+0.64\left[\frac{h}{b} \arctan \left(\frac{h}{b}\right)\right]\right\} K_{r} \tan \phi \frac{K_{\omega}}{2 i l}\left[\frac{2\left(e^{1-\frac{i l}{K_{\omega}}}-e+e^{-\frac{i l}{K_{\omega}}}-1\right)}{1+\frac{4 n_{p}{ }^{2} \pi^{2} K_{\omega}{ }^{2}}{i^{2} l^{2}}}+\frac{e^{1-\frac{2 i l}{K_{\omega}}}-e}{1+\frac{2 n_{p}{ }^{2} \pi^{2} K_{\omega}{ }^{2}}{i^{2} l^{2}}}\right]
\end{aligned}
$$

\subsection{Compaction resistance}

Rowland used the mean value of the maximum pressures generated beneath each wheel [18], instead of the nominal uniform pressure, to estimate the sinkage and compaction resistance. The mean maximum pressure can be computed as:

$$
p_{m m}=\frac{1.25 W}{2 n_{w} b \sqrt{p_{t} d_{w}}}
$$

where $W$ is the vehicle weight, $n_{w}$ is the number of road wheels per track, $b$ is the track width, $d_{w}$ is the outer diameter of the road wheel and $p_{t}$ is the track pitch.

Substituting $p=p_{m m}$ in Eq. (1) gives a sinkage of:

$$
z=b\left(\frac{1.25 W}{2\left(k_{c}+b k_{\phi}\right) n_{w} b \sqrt{p_{t} d_{w}}}\right)^{1 / n}
$$

From energy considerations, the total work done by compressing the soil can be expressed as:

$$
E=b \int_{0}^{l} \int_{0}^{z} p_{m m} d l d z=b l \int_{0}^{z}\left(\frac{k_{c}}{b}+k_{\phi}\right) z^{n} d z=b l\left(\frac{k_{c}}{b}+k_{\phi}\right)\left(\frac{z^{n+1}}{n+1}\right)
$$

Substituting Eq. (8) into Eq. (9), with $E=R_{c} l$, gives the compaction resistance as: 


$$
R_{c}=\frac{b}{(n+1)\left(k_{c} / b+k_{\phi}\right)^{1 / n}}\left(\frac{1.25 W}{2 n_{w} b \sqrt{p_{t} d_{w}}}\right)^{\frac{n+1}{n}}
$$

\subsection{Bulldozing resistance}

The bulldozing resistance, a result of pushing the sediment in front of the tracks of the miner, should be considered and can be computed using passive earth pressure theory [17]:

$$
R_{b}=2 b\left(0.5 \gamma_{s} h^{2} K_{\gamma}+h q K_{q}+h c K_{c}\right)
$$

where $\gamma_{s}$ is the specific weight of the sediment; $h$ is the grouser height; and $K_{\gamma}=\left(2 N_{\gamma}, \tan \phi+1\right) \cos ^{2} \phi, K_{q}=N_{q} \cos ^{2} \phi$ and $K_{c}=\left(N_{c}-\tan \phi\right) \cos ^{2} \phi$, where $N_{\gamma}, N_{q}$ and $N_{c}$ are the coefficients of the Terzaghi bearing capacity determined by the soil internal friction angle $\phi$. As $\phi$ is approximately 5 degrees for sediments, and $N_{\gamma}=0.51, N_{q}=0.62$ and $N_{c}=7.32$, can be approximately determined.

\subsection{Net traction force}

As an application of the above-derived equations, the net traction force for the miner on the seafloor can finally be computed as:

$$
\begin{aligned}
& F(i)=F_{s}(i)-R_{c}-R_{b} \\
& =\left\{(A+4 h l) c+W \tan \phi\left[1+0.64 \frac{h}{b} \arctan \left(\frac{h}{b}\right)\right]\right\} K_{r}\left\{1+\frac{K_{\omega}}{i l}\left[e^{-\left(i l / K_{\omega}\right)}-1\right]\left[-\left[\frac{e}{K_{r}(e-1)}-1\right] \frac{K_{\omega}}{2 i l}\left[2 e^{1-\left(i l / K_{\omega}\right)}-e^{1-\left(2 i l / K_{\omega}\right)}-e\right]\right\}\right. \\
& -W\left\{1+0.64\left[\frac{h}{b} \arctan \left(\frac{h}{b}\right)\right]\right\} K_{r} \tan \phi \frac{K_{\omega}}{2 i l}\left[\frac{2\left(e^{1-\frac{i l}{K_{\varphi}}}-e+e^{-\frac{i l}{K_{K^{\prime}}}}-1\right)}{1+\frac{4 n_{p}{ }^{2} \pi^{2} K_{\omega}{ }^{2}}{i^{2} l^{2}}}+\frac{e^{1-\frac{2 i l}{K_{\omega}}-e}}{1+\frac{2 n_{p}{ }^{2} \pi^{2} K_{\omega}{ }^{2}}{i^{2} l^{2}}}\right]-\frac{2 b}{(n+1)\left(\frac{k_{c}}{b}+k_{\phi}\right)}\left(\frac{1.25 W}{2 n_{w} b \sqrt{p_{t} d_{\omega}}}\right)^{\frac{n+1}{n}}-2 b\left[\frac{1}{2} \gamma_{s} h^{2} K_{\gamma}+h\left(q K_{q}+c K_{c}\right)\right]
\end{aligned}
$$

Substituting the parameters given in Tables I and II into Eq. (12), gives the net traction force on the seafloor sediment, which is illustrated as a solid line in Fig. 12. For comparison, the traction force on a land surface of common soil is presented in Fig. 12 as a dashed line.

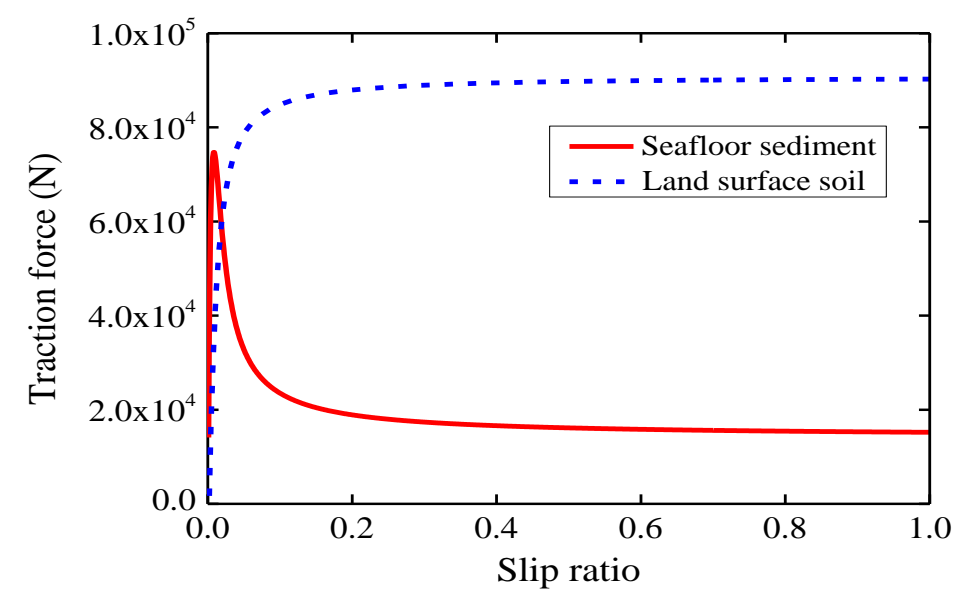

Figure 12: Traction force-slip ratio relationship curves for the miner on seafloor sediment and on a land surface of common soil.

The traction force on the seafloor sediment initially increases rapidly and reaches a maximum of approximately $7.5 \times 10^{4} \mathrm{~N}$ at an optimum slip ratio of approximately $3 \%$; then, it decreases rapidly and approaches a nearly constant residual value of approximately $2.0 \times 10^{4} \mathrm{~N}$ for further slip increase. By contrast, on the land surface soil, the traction force continues to 
increase until the slip ratio is approximately $10 \%$ and then remains at a nearly constant value with further slip increase.

\section{CONCLUSIONS}

The major conclusions that can be drawn from this work are listed below.

(1) Mathematical relationships for the pressure-sinkage and shear stress-displacement between the seafloor tracked miner and the seafloor sediment have been obtained. The shear property of the seafloor sediment exhibits a completely different pattern from that of land surface common soils in that it initially increases rapidly and reaches a maximum at a particular shear displacement of approximately $0.035 \mathrm{~m}$ and then decreases and approaches a nearly constant residual value. The ratio of residual shear stress to maximum shear stress is equal to approximately 0.28 .

(2) An innovative user-written subroutine for characterizing the special characteristics of the seafloor sediment has been developed and linked into the RecurDyn simulation environment, based on which a new 3D multi-body simulation model of the tracked miner has been developed. Simulations under various conditions prove that the miner has good geometrical trafficability, bearing trafficability and locomotion performance on the seafloor.

(3) A mathematical model for evaluating the tractive trafficability of a miner on the seafloor has been developed. The model reveals a special property. The traction force initially increases rapidly with the slip ratio, displaying the maximum traction force at the optimum slip ratio of $3 \%$. The traction force then decreases to a nearly constant residual value at a slip ratio of approximately $20 \%$. As the traction force will rapidly decrease at slips higher than the optimum slip, a lack of traction force or serious sinkage will be more likely on seafloor sediments.

\section{ACKNOWLEDGEMENTS}

This research is supported by National Deep-sea Technology Project of Development and Research of China (Grant No. DYXM-115-04-02-01), National High Technology Research and Development Program (Grand No. 2012AA091201), Knowledge Innovation Foundation Research Project of Shenzhen Science and Technology Program (JCYJ20130401160614378), and the Natural Science Foundation of Hunan Province (13JJ4021). The authors would like to thank the comments from the anonymous reviewers that improved the quality of the paper.

\section{REFERENCES}

[1] Schulte, E.; Handschuh, R; Schwarz, W. (2003). Transferability of soil mechanical parameters to traction potential calculation of a tracked vehicle, Proceedings of the $5^{\text {th }}$ ISOPE Ocean Mining Symposium, 123-131

[2] Schulte, E.; Schwarz, W. (2009). Simulation of tracked vehicle performance on deep sea soil based on soil mechanical laboratory measurements in bentonite soil, Proceedings of the $8^{\text {th }}$ ISOPE Ocean Mining Symposium, 276-284

[3] Yamazaki, T.; Komine, T.; Kawakami, T. (2005). Geotechnical properties of deep-sea sediments and the in-situ measurement techniques, Proceedings of the $6^{\text {th }}$ ISOPE Ocean Mining Symposium, 48-55

[4] Choi, J.-S.; Hong, S.; Chi, S.-B.; Lee, H.-B.; Park, C.-K.; Kim, H.-W.; Yeu, T.-K.; Lee, T. H. (2011). Probability distribution for the shear strength of seafloor sediment in the KR5 area for the development of manganese nodule miner, Ocean Engineering, Vol. 38, No. 17-18, 2033-2041, doi:10.1016/j.oceaneng.2011.09.011 
[5] Choi, J. S.; Hong, S.; Kim, H. W. (2003). An experimental study on tractive performance of tracked vehicle on cohesive soft soil, Proceedings of the $5^{\text {th }}$ ISOPE Ocean Mining Symposium, 139-143

[6] Kim, H.-W.; Hong, S.; Choi, J.-S.; Yeu, T.-K. (2006). Dynamic analysis of underwater tracked vehicle on extremely soft soil by using Euler parameters, Journal of Ocean Engineering and Technology, Vol. 20, No. 6, 93-100

[7] Kim, H.-W.; Lee, C.-H.; Hong, S.; Oh, J.-W.; Min, C.-H.; Yeu, T.-K.; Choi, J. (2013). Dynamic analysis of a tracked vehicle based on a subsystem synthesis method, Proceedings of the $10^{\text {th }}$ ISOPE Ocean Mining Symposium, 279-285

[8] Dai, Y.; Liu, S.-J. (2013). An integrated dynamic model of ocean mining system and fast simulation of its longitudinal reciprocating motion, China Ocean Engineering, Vol. 27, No. 2, 231-244, doi:10.1007/s13344-013-0020-1

[9] Rubinstein, D.; Hitron, R. (2004). A detailed multi-body model for dynamic simulation of off-road tracked vehicles, Journal of Terramechanics, Vol. 41, No. 2-3, 163-173, doi:10.1016/ j.jterra.2004.02.004

[10] Rubinstein, D.; Coppock, J. L. (2007). A detailed single-link track model for multi-body dynamic simulation of crawlers, Journal of Terramechanics, Vol. 44, No. 5, 355-364, doi:10.1016/ j.jterra.2007.10.004

[11] Gao, Y.; Wong, J. Y. (1994). The development and validation of a computer aided method for design evaluation of tracked vehicles with rigid links, Proceedings of the Institution of Mechanical Engineers, Part D: Journal of Automobile Engineering, Vol. 208, No. 3, 207-215, doi:10.1243/PIME_PROC_1994_208_183_02

[12] Wong, J. Y.; Huang, W. (2005). Evaluation of the effects of design features on tracked vehicle mobility using an advanced computer simulation model, International Journal of Heavy Vehicle Systems, Vol. 12, No. 4, 344-365, doi:10.1504/IJHVS.2005.008304

[13] Wong, J. Y.; Senatore, C.; Jayakumar, P.; Iagnemma, K. (2015). Predicting mobility performance of a small, lightweight track system using the computer-aided method NTVPM, Journal of Terramechanics, Vol. 61, 23-32, doi:10.1016/j.jterra.2015.07.002

[14] Janarthanan, B.; Padmanabhan, C.; Sujatha, C. (2012). Longitudinal dynamics of a tracked vehicle: Simulation and experiment, Journal of Terramechanics, Vol. 49, No. 2, 63-72, doi:10.1016/j.jterra.2011.11.001

[15] Bekker, M. G. (1969). Introduction to Terrain-Vehicle Systems, University of Michigan Press, Ann Arbor

[16] Janosi, Z.; Hanamto, B. (1961). The analytical determination of drawbar pull as a function of slip for tracked vehicles in deformable soils, $I^{s t}$ International Conference of the International Society for Terrain-Vehicle Systems, 707-736

[17] Wong, J. Y. (2010). Terramechanics and Off-Road Vehicle Engineering, Elsevier Science Publishers, Amsterdam

[18] Rowland, D. (1975). A review of vehicle design for soft-ground operation, Proceedings of the $5^{\text {th }}$ International Conference of the International Society for Terrain-Vehicle System, 179-219 